\title{
Short-Term Outcomes of Laparoscopic/Robotic Gastrectomy Compared with Open Gastrectomy for Advanced Gastric Cancer Following Chemotherapy
}

Kozo Yoshikawa ( $\square$ yoshikawa.kozo@tokushima-u.ac.jp )

The University of Tokushima

Mitsuo Shimada

The University of Tokushima

Takuya Tokunaga

The University of Tokushima

Toshihiro Nakao

The University of Tokushima

\section{Masaaki Nishi}

The University of Tokushima

Chie Takasu

The University of Tokushima

Hideya Kashihara

The University of Tokushima

\section{Yuma Wada}

The University of Tokushima

Toshiaki Yoshimoto

The University of Tokushima

Shoko Yamashita

The University of Tokushima

\section{Research Article}

Keywords: Chemotherapy, Laparoscopic/robotic gastrectomy, Short-term outcome

Posted Date: December 22nd, 2021

DOI: https://doi.org/10.21203/rs.3.rs-1140557/v1

License: (c) (1) This work is licensed under a Creative Commons Attribution 4.0 International License.

Read Full License 


\section{Abstract \\ Purpose}

This study aimed to investigate the short-term outcomes of laparoscopic gastrectomy/robotic gastrectomy after chemotherapy in patients with advanced gastric cancer and compare these outcomes with those of open gastrectomy.

\section{Methods}

Fifty patients who underwent radical gastrectomy for advanced gastric cancer after chemotherapy between 2007 and 2021 were retrospectively analyzed. The patients were divided into two groups: the laparoscopic gastrectomy/robotic gastrectomy $(n=11)$ and open gastrectomy $(n=39)$ groups. The short-term outcomes of these procedures were subsequently examined.

\section{Results}

The laparoscopic gastrectomy/robotic gastrectomy group had significantly shorter hospital stays and lower intraoperative blood loss than the open gastrectomy group. The overall complication rates were $12.8 \%$ ( 5 of 39 patients) and $0 \%$ ( 0 of 11 patients) in the open gastrectomy and laparoscopic gastrectomy/robotic gastrectomy groups, respectively $(P=0.1)$.

\section{Conclusions}

Laparoscopic gastrectomy/robotic gastrectomy may be a surgical option after chemotherapy for patients with advanced gastric cancer.

\section{Background}

Globally, gastric cancer is the third leading cause of cancer death, with $>70 \%$ of the cases reported in Asian populations, and it remains a major health problem [1,2,3]. Furthermore, long-term outcomes in advanced cases remain unsatisfactory. Neoadjuvant chemotherapy (NAC) has been increasingly used in the treatment of locally advanced gastric cancer [4] and is generally accepted in Western countries based on the results of the FLOT4 and MAGIC trials $[5,6]$. In Japan, however, NAC is recommended for only locally advanced cases with local metastasis, and gastrectomy with D2 lymph node dissection and adjuvant chemotherapy is the standard treatment for advanced gastric cancer [3]. Stage IV gastric cancer is categorized into four groups, category 1 and 2 patients have a chance to be performed curative surgery. [7] and owing to advances in chemotherapy, resection of localized unresected gastric cancer has become possible, and the conversion rate is gradually increasing [8]. 
Regarding the surgical approach, laparoscopic gastrectomy (LG), a minimally invasive surgery, is recommended for early stages of gastric cancer. Recently, the usefulness of LG for advanced gastric cancer has been reported and have confirmed the efficacy and safety of LG for advanced gastric cancer [3]. Furthermore, the CLASS-01 trial, which was a phase III study [9], suggested that, ontologically, LG was safe and comparable to open gastrectomy (OG). Recently, robotic gastrectomy (RG), which can be used to perform more sensitive surgeries compared with LG, has become more prevalent [10]. The feasibility of RG for advanced gastric cancer has become gradually accepted and the complications of this type of surgery have been reported to be similar to those of LG.

Chemotherapy-induced tissue fibrotic changes and edema lead to technical difficulties in laparoscopic procedures. Few studies have compared the safety and efficacy of OG and LG/RG following NAC and conversion surgery in patients with advanced gastric cancer. Therefore, the indication of LG/RG in such patients remains controversial.

The aim of this study was to investigate the short-term outcomes of LG/RG in patients with advanced gastric cancer who underwent chemotherapy and evaluate its feasibility as a treatment for advanced gastric cancer compared with OG.

\section{Methods}

\section{Patients}

We performed a retrospective analysis of the gastric cancer database at the Department of Surgery, Tokushima University, Japan. Between October 2007 and February 2021, 64 patients were diagnosed with advanced gastric cancer and underwent preoperative chemotherapy followed by gastrectomy. The indications for NAC were large type 3 (>80 mm) or type 4 advanced gastric cancer or bulky lymph node metastasis. Patients with unresectable gastric cancer had undergone chemotherapy. The indication of the conversion surgery is that the $\mathrm{CT}$ and gastric fiber reveals that RO surgery can be performed. NAC regimens and regimens for unresectable gastric cancer mainly consisted of docetaxel+cisplatin+TS-1 (DCS), TS-1+cisplatin, or TS-1+oxaliplatin. Patients with human epidermal growth factor 2 (HER2)positive gastric cancer also received trastuzumab. Patients who underwent palliative gastrectomy $(n=$ 14) were excluded from the present study. Fifty patients were finally selected and subsequently divided into two groups: the $L G / R G$ group $(n=11)$ and the OG group $(n=39)$. All patients had undergone radical gastrectomy according to the treatment guidelines of the Japanese Gastric Cancer Association (JGCA) [11]. All LG/RG procedures were performed by qualified surgeons certified by the Endoscopic Surgical Skill Qualification System (ESSQS) of the Japan Society for Endoscopic Surgery (JSES). This study was approved by the Tokushima University Review Board.

\section{Operative technique}

All surgical procedures were performed in accordance with the guidelines of JGCA [11]. Total or distal gastrectomy was performed in accordance with the location of the primary tumor. During total 
gastrectomy, splenectomy was performed in patients for whom the tumor invaded the upper one-third of the greater curvature of the stomach. The reconstruction method used in both groups comprised either the Billroth I or Roux-en-Y(R-Y) procedure depending on the remnant volume of the stomach and the extent of tumor invasion in the duodenum. The selection of $L G / R G$ or $O G$ was based on the decision made during departmental conference. In the LG/RG group, reconstruction was performed via intracorporeal anastomosis. During intracorporeal reconstruction, R-Y gastrojejunostomy and deltashaped Billroth I anastomosis were performed in patients undergoing distal gastrectomy.

Esophagojejunostomy was performed using the circular method. In the OG group, Billroth I anastomosis was performed via hand suturing. R-Y gastrojejunostomy was performed via functional side-to-side anastomosis. In total gastrectomy, R-Y esophagojejunostomy was performed using the circular method.

\section{Data collection}

Clinicopathological parameters and perioperative data (age, body mass index, American Society of Anesthesiologists (ASA) score [12], intraoperative blood loss, operative time, postoperative complications, length of postoperative hospital stay, clinicopathological TNM stage, and tumor regression grade) were evaluated according to the JGCA classification of gastric carcinoma [13]. Postoperative complications were defined according to the Clavien-Dindo classification system, and only those of grade 3 or higher were recorded [14]. Hospital mortality was defined as death from any cause within the postoperative 30 days.

\section{Statistical analyses}

Data were analyzed using the JMP statistical software program (SAS Institute Inc., Cary, NC). Patient characteristics were compared using the $\chi^{2}$ test. Quantitative variables are presented as means \pm standard deviation. A P-value of $<0.05$ was considered statistically significant.

\section{Ethics approval and consent to participant}

This study was designed in accordance with the Declaration of Helsinki and was approved by the institutional review board of Tokushima University. All patients received sufficient explanation of the study and written informed consent was obtained.

\section{Results}

The median ages of the patients in the $O G$ and LG/RG groups were 65.8 and 70.4 years, respectively. ASA score of 1 was more frequent in the OG group. The tumor diameter was larger in the OG group than in the LG/RG group (53.5 vs. $27.6 \mathrm{~mm}, \mathrm{P}<0.01$ ). The most frequent regimen was DCS in both groups. The pathological stage was higher in the OG group, whereas the pathological response was higher in the LG/RG group. The indication of preoperative chemotherapy was similar between the two groups (Table 1). 
Table 1

Patient's characteristics

\begin{tabular}{|c|c|c|c|}
\hline & OG group & LG/RG group & $P$ value \\
\hline & $(n=39)$ & $(n=11)$ & \\
\hline Age (years) & $65.8 \pm 1.5$ & $70.4 \pm 2.8$ & 0.15 \\
\hline Sex & & & 0.95 \\
\hline Male & 28 & 8 & \\
\hline Female & 11 & 3 & \\
\hline BMI & $21.2 \pm 0.5$ & $21.9 \pm 0.9$ & 0.49 \\
\hline ASA & & & 0.03 \\
\hline 1 & 24 & 2 & \\
\hline 2 & 14 & 8 & \\
\hline 3 & 1 & 1 & \\
\hline Tumor size (mm) & $53.5 \pm 4.9$ & $27.6 \pm 9.3$ & 0.01 \\
\hline Histologiccal type & & & 0.28 \\
\hline Differentiated & 11 & 5 & \\
\hline Undefferentiated & 28 & 6 & \\
\hline Regimen & & & 0.12 \\
\hline DCS/DCS-Tmab & 28 & 6 & \\
\hline S1+ipPTX & 4 & 1 & \\
\hline SP/SOX/SOX-Tmab & 7 & 4 & \\
\hline Total cycle of chemothrapy & $4.1 \pm 0.4$ & $6.6 \pm 0.8$ & $<0.01$ \\
\hline Pathological stage & & & 0.01 \\
\hline No tumor & 4 & 2 & \\
\hline I & 4 & 3 & \\
\hline ॥ & 15 & 6 & \\
\hline III & 16 & 0 & \\
\hline Pathological response* & & & 0.01 \\
\hline Grade 0 & 3 & 2 & \\
\hline
\end{tabular}




\begin{tabular}{|c|c|c|c|}
\hline & $O G$ group & LG/RG group & $P$ value \\
\hline Grade 1 & 20 & 0 & \\
\hline Grade 2 & 7 & 6 & \\
\hline Grade 3 & 5 & 3 & \\
\hline Operation & & & 0.1 \\
\hline NAC & 8 & 5 & \\
\hline Conversion & 31 & 6 & \\
\hline \multicolumn{4}{|c|}{ OG: Open gastrectomy } \\
\hline \multicolumn{4}{|c|}{ LG: Laparoscopic gastrectomy } \\
\hline \multicolumn{4}{|c|}{ RG: Robotic gastrectomy } \\
\hline \multicolumn{4}{|c|}{ BMI: Body mass index } \\
\hline \multicolumn{4}{|c|}{ ASA: Amerian social of Anesthesiologists } \\
\hline \multicolumn{4}{|c|}{ DCS: Docetaxel+Cisplatin+S-1 } \\
\hline \multicolumn{4}{|c|}{ ipPTX: Intraperitoneal Paclitaxel } \\
\hline \multicolumn{4}{|c|}{ SP: S-1+Cisplatin } \\
\hline \multicolumn{4}{|c|}{ SOX: S-1+Oxaliplatin } \\
\hline \multicolumn{4}{|c|}{ NAC: Neoadjuvant chemotherapy } \\
\hline *: According & Classificatic & of Gastric Ca & \\
\hline
\end{tabular}

The surgical data of the two groups are summarized in Table 2. There were significant differences in the procedure of gastrectomy between the two groups: in the OG group, total gastrectomy was more frequently performed; therefore, $\mathrm{R}-\mathrm{Y}$ reconstruction was more frequently implemented. The operative time tended to be longer in the LG/RG group compared with the OG group (304 vs. $273 \mathrm{~min}, \mathrm{P}=0.29$ ). There was a significant difference in the intraoperative blood loss between the two groups: blood loss was lower in the LG/RG group than in the OG group (60 vs. $153 \mathrm{ml}, \mathrm{P}=0.04$ ). Further, in the LG/RG group, D2 lymph node dissection was performed in all patients. No patients in the LG/RG group required switching to open surgery. There was no significant difference in the number of dissected lymph nodes between the two groups (30 vs. $28 \mathrm{P}=0.69$ ). 
Table 2

Intraoperative outcomes

\begin{tabular}{|llll|}
\hline & OG group & LG/RG group & P value \\
\hline Type of gastrectomy & $(\mathrm{n}=39)$ & $(\mathrm{n}=11)$ & \\
\hline Distal gastrectomy & & & 0.05 \\
\hline Total gastrectomy & 9 & 6 & \\
\hline Lymph node dissection & 30 & 5 & 0.21 \\
\hline D1+ & & & \\
\hline D2 & 3 & 0 & 0.47 \\
\hline Splenectomy & 36 & 11 & 0.72 \\
\hline Liver metastatic resection & 1 & 0 & $<0.01$ \\
\hline Reconstruction & 5 & 1 & \\
\hline Billtorh-I & & & \\
\hline Roux-en-Y & 2 & 6 & 0.29 \\
\hline Operation time & 37 & 5 & 0.04 \\
\hline Intraoperative blood loss & $273 \pm 13$ & $304 \pm 25$ & \\
\hline The number of disseted lymph nodes & $30 \pm 1.9$ & $28 \pm 3.6$ & 0.69 \\
\hline OG: Open gastrectomy & & & \\
\hline LG: Laparoscopic gastrectomy & & & \\
\hline RG: Robotic gastrectomy & & & \\
\hline
\end{tabular}

The postoperative factors are presented in Table 3. The overall complication rates were $12.8 \%$ ( 5 of 39 patients) and $0 \%$ ( 0 of 11 patients) in the $O G$ and $L G / R G$ groups, respectively $(P=0.1)$. The surgical complications included anastomotic leakage $(n=2)$, pancreatic fistula $(n=1)$, ascites $(n=1)$, and chylothorax $(n=1)$. In the OG group, one patient required reoperation owing to hemothorax and hemorrhage. The LG/RG group had a significantly shorter length of postoperative hospital stay than the OG group (10.1 vs. 16.7 days, $P=0.04$ ). Hospital mortalities were not observed in either group. 
Table 3

Postoperative outcomes

\begin{tabular}{|llll|}
\hline & OG group & LG/RG group & P value \\
\hline & $(\mathrm{n}=39)$ & $(\mathrm{n}=11)$ & \\
\hline Complication CD grade $\geq 3$ & & & 0.1 \\
\hline Overall & 5 & 0 & \\
\hline Pancreatic fistula & 1 & 0 & \\
\hline Anastomotic leakage & 2 & 0 & 0.04 \\
\hline Ascites & 1 & 0 & \\
\hline Chylothorax & 1 & 0 & \\
\hline Requiring re-operation & 1 & 0 & \\
\hline Post operative hospital stay & $16.7 \pm 1.5$ & $10.1 \pm 2.8$ & \\
\hline Hospital mortality & 0 & 0 & \\
\hline CD: Clavien-Dindo & & & \\
\hline
\end{tabular}

\section{Discussion}

This study retrospectively investigated the surgical outcomes of $L G / R G$ following chemotherapy and compared them with those of OG. The results showed that LG/RG following chemotherapy is safe and feasible owing to its good short-term outcomes.

The development of new anticancer drugs and highly effective regimens has enabled remarkable tumor shrinkage with chemotherapy, whereby some patients with stage IV gastric cancer demonstrating a good response to chemotherapy have been able to undergo curative resection followed by long-term survival [15]. The current literature demonstrates that the so-called conversion surgery for unresectable stage III or stage IV gastric cancer is associated with longer survival than chemotherapy alone. On the other hand, NAC has been proven to increase the R0 resection rate and reduce lymph node metastases compared with surgery alone [16]. The European Organization for Research and Treatment of Cancer randomized trial illustrated that the RO dissection rate in the NAC group was significantly higher than that in the surgery-alone group and that the NAC group had fewer lymph node metastases [17].

LG is accepted as being more effective than conventional open surgery and is commonly used to treat clinical stage I gastric cancer in accordance with the recent technical and instrumental improvements. Even for locally advanced gastric cancer, the technical safety of $L G$ has been demonstrated in a randomized phase II study. A large phase III trial, KLASS-02[18], reported that LG with D2 lymphadenectomy was associated with lower postoperative complication rates, faster recovery, and less 
pain compared with OG. Moreover, laparoscopic surgery also offers benefits in gastrectomy for locally advanced gastric cancer after neoadjuvant chemotherapy, such as better postoperative safety and adjuvant chemotherapy tolerance, compared with conventional open surgery. A randomized controlled study conducted in east Asia confirmed that the technical feasibility of gastrectomy with D2 lymphadenectomy performed by laparoscopy was comparable to that of OG [19]. For patients with advanced gastric cancer who have not undergone preoperative chemotherapy, RG with D2 lymphadenectomy is feasible and safe for the treatment of advanced gastric cancer in terms of the lower incidence and severity of its complications [10]. Robot-assisted D2 gastrectomy is also technically reasonable, although its long-term outcomes are yet to be evaluated in prospective studies in Japan.

In the current study, patients in the LG/RG group had significantly lower intraoperative blood loss and better postoperative recovery compared with the OG group, with previous studies reporting similar findings for patients with advanced gastric cancer who had not undergone NAC and conversion surgery $[3,20]$. The findings of the current study show that the advantages of LG/RG remain the same for patients with advanced gastric cancer who undergo preoperative chemotherapy. The precise operative techniques involved in $L G / R G$ help to minimize intraoperative blood loss. Because intraoperative blood loss is associated with the prognosis of gastric cancer $[21,22,23]$. LG/RG may help improve the prognosis for some patients.

No significant differences were observed between the postoperative complication rates in the LG/RG ( 0 patients, $0 \%$ ) and $O G$ groups (5 patients, $13 \%)(P=0.1)$ in this study, a finding which was consistent with the morbidity rates about $10 \%$ reported in previous studies on patients who underwent open D2 gastrectomy following NAC and conversion $[3,8]$. The incidence of surgical complications in the LG/RG group can be considered low.

Chemotherapy causes tissue fibrosis, tissue edema and necrosis and destroy the anatomical dissection plane. As the result, these reactions may interfere with the surgical procedure and makes surgery more difficult, therefore, increase postoperative complication rates. Therefore, more operative blood loss has been observed in patients who have previously undergone neoadjuvant therapy, in whom it was harder and more tedious to stop the bleeding. D2 lymphadenectomy after neoadjuvant chemotherapy is more risky than D2 lymphadenectomy without preoperative treatment. Such issues may be resolved by LG/RG because this procedure allows for visual magnification, better exposure, and more delicate maneuvers of organs, vessels, and nerves [24,25]. Operative hemorrhage can be easily induced by fibrosis and tissue edema, although an ultrasonic knife plays an important role in hemostasis. No postoperative complications and mild intraoperative bleeding were observed in this study. Although the operative time tends to be longer, $L G / R G$ should be considered for patients who have undergone preoperative chemotherapy.

Previously, patients who received preoperative chemotherapy underwent open surgery. However, as laparoscopic and robotic surgery became increasingly adopted, the indication for LG/RG was expanded to pretreatment cases. All procedures were performed by qualified surgeons certified by the ESSQS of the 
JSES who had adequate experience. It is therefore difficult to state that RG/LG itself is effective in all cases of preoperative chemotherapy. LG performed by credentialed surgeons was surgically safe and feasible for patients with advanced gastric cancer compared with conventional OG [26].

This study had several limitations. First, the indications of the preoperative chemotherapy were different for patients undergoing NAC and conversion surgery. Hence, these two should be investigated individually in the future. This study focused on short-term results based on the surgical method; therefore, both patients undergoing NAC and conversion surgery were included. Other limitations of this study were the limited sample size, the retrospective nature, and the lack of randomization in the two treatment arms. Finally, only the short-term outcomes were examined, and the long-term outcomes remain to be investigated.

\section{Conclusions}

In conclusion, the findings of this study revealed that $L G / R G$ following chemotherapy for advanced gastric cancer has good short-term outcomes, which suggests that $L G / R G$ is a good option for patients with gastric cancer who have undergone chemotherapy.

\section{Abbreviations}

NAC: Neoadjuvant chemotherapy

LG: laparoscopic gastrectomy

OG: open gastrectomy

RG: robotic gastrectomy

DCS: docetaxel+cisplatin+TS-1

HER2: human epidermal growth factor 2

JGCA: Japanese Gastric Cancer Association

ESSQS: Endoscopic Surgical Skill Qualification System

JSES: Japan Society for Endoscopic Surgery

\section{Declarations}

\section{Consent for publication}

Not applicable 
Availability of data and materials

The datasets used during the current study are available from the corresponding author on reasonable request.

\section{Competing interests}

The authors declare that they have no competing interests.

\section{Funding}

There is no funding to be declared

\section{Authors' contribution}

$\mathrm{KY}$ and MS designed the research, $\mathrm{KY}, \mathrm{TT}$, and TN analyzed the data. KY, MN, CT, HK, YW, TY, and SY collected clinical data and helped to write the manuscript. All authors read and approved the final manuscript.

\section{Acknowledgements}

None

\section{References}

1. Kinoshita J, Yamaguchi T, Moriyama H, Fushida S. Current status of conversion surgery for stage IV gastric cancer. Surg Today. 2021;51:1736-1754.

2. Kodera Y. Surgery with curative intent for stage IV gastric cancer: Is it a reality of illusion? Ann Gastroenterol Surg. 2018;2:339-347.

3. Fujisaki M, Mitsumori N, Shinohara T, Takahashi N, Aoki H, Nyumura Y, et al. Short- and long-term outcomes of laparoscopic versus open gastrectomy for locally advanced gastric cancer following neoadjuvant chemotherapy. Surg Endosc. 2021;35:1682-1690.

4. Xing J, Wang Y, Shan F, Li S, Jia Y, Ying X, et al. Comparison of totally laparoscopic and laparoscopic assisted gastrectomy after neoadjuvant chemotherapy in locally advanced gastric cancer. Eur J Surg Oncol. 2021;6:S0748-7983.

5. Cunningham D, Allum WH, Stenning SP, Thompson JN, Van de Velde CJ, Nicolson M, et al. Perioperative chemotherapy versus surgery alone for respectable gastroesophageal cancer. $\mathrm{N}$ Engl $\mathrm{J}$ Med. 2006;355:11-20.

6. Al-Batran SE, Homann N, Pauligk C, Goetze TO, Meiler J, Kasper S, et al. Investigators erioperative chemotherapy with fluorouracil plus leucovorin, oxaliplatin, and docetaxel versus fluorouracil or capecitabine plus cisplatin and epirubicin for locally advanced, resectable gastric or gastro- 
oesophageal junction adenocarcinoma (FLOT4):a randomized, phase 2/3 trial. Lancet. 2019;393:1948-1957.

7. Yoshida K, Yamaguchi K, Okumura N, Tanahashi T, Kodera Y. Is conversion therapy possible in stage IV gastric cancer: the proposal of new biological categories of classification. Gastric Cancer. 2016;19:329-338.

8. Yamamoto K, Omori T, Hara H, Shinno N, Sugimura K, Miyata $\mathrm{H}$, et al. Minimally invasive surgery is feasible after preoperative chemotherapy for stage IV gastric cancer. Ann Gastroenterol Surg. 2020;4:396-404.

9. Park YK, Yoon HM, Kim YW, Park JY, Ryu KW, Lee YJ, et al. Laparoscopy-assisted versus open D2 distal gastrectomy for advanced gastric cancer: results from a randomized phase II multicenter clinical trial (COACT 1001). Ann Surg. 2018;267:638-645.

10. Wang WJ, Li HT, Yu JP, Su L, Guo CA, Chen P, et al. Severity and incidence of complications assessed by the Clavien-Dindo classification following robotic and laparoscopic gastrectomy for advanced gastric cancer: a retrospective and propensity score-matched study. Surg Endosc. 2019;33:33413354.

11. Japanese Gastric Cancer Association Japanese gastric cancer treatment guidelines 2018 (5th edition). Gastric Cancer. 2021;24:1-21.

12. Charlson ME, Pompei P, Ales KL, MacKenzie CR. A new method of classifying prognostic comorbidity in longitudinal studies: development and validation. J Chronic Dis. 1987;40:373-383.

13. Japanese Gastric Cancer Association Japanese gastric cancer treatment guidelines 2010 (ver. 3). Gastric Cancer. 2011;14:113-123.

14. Dindo D, Demartines N, Clavien PA Classification of surgical complications: a new proposal with evaluation in a cohort of 6336 patients and results of a survey. Ann Surg. 2004;240:205-213.

15. Solaini L, Ministrini S, Bencivenga M, D'Ignazio A, Marino E, Cipollari C, et al. Conversion gastrectomy for stage IV unresectable gastric cancer: a GIRCG retrospective cohort study. Gastric Cancer. 2019;22:1285-1293.

16. Li Z, Shan F, Wang Y, Li S, Jia Y, Zhang L, et al. Laparoscopic versus open distal gastrectomy for locally advanced gastric cancer after neoadjuvant chemotherapy: safety and short-term oncologic results. Surg Endosc. 2016;30:4265-71.

17. Schuhmacher C, Gretschel S, Lordick F, Reichardt P, Hohenberger W, Eisenberger CF, et al. Neoadjuvant chemotherapy compared with surgery alone for locally advanced cancer of the stomach and cardia: European Organization for Research and Treatment of Cancer randomized trial 40954. J Clin Oncol. 2010;28:5210-8.

18. Kim MC, Kim W, Kim HH, Ryu SW, Ryu SY, Song KY, et al. Risk factors associated with complication following laparoscopy-assisted gastrectomy for gastric cancer: a large-scale Korean multicenter study. Ann Surg Oncol. 2008;15:2692-2700.

19. Tokunaga M, Sato $Y$, Nakagawa M, Aburatani T, Matsuyama T, Nakajima $Y$, et al. Perioperative chemotherapy for locally advanced gastric cancer in Japan: current and future perspectives. Surg 
Today. 2020;50:30-37.

20. Yasufuku I, Nunobe S, Ida S, Kumagai K, Ohashi M, Hiki N, et al. Conversion therapy for peritoneal lavage cytology-positive type 4 and large type 3 gastric cancer patients selected as candidates for R0 resection by diagnostic staging laparoscopy. Gastric Cancer. 2020;23:319-327.

21. Mörner ME, Gunnarsson U, Jestin P, Svanfeldt M The importance of blood loss during colon cancer surgery for long- term survival: an epidemiological study based on a population based register. Ann Surg. 2012;255:1126-1128.

22. Nagai S, Fujii T, Kodera Y, Kanda M, Sahin TT, Kanzaki A, et al. Impact of operative blood loss on survival in invasive ductal adenocarcinoma of the pancreas. Pancreas. 2011;40:3-9.

23. Liang YX, Guo HH, Deng JY, Wang BG, Ding XW, Wang XN, et al. Impact of intraoperative blood loss on survival after curative resection for gastric cancer. World J Gastroenterol. 2013;19:5542-5550.

24. Feng $\mathrm{D}$, Leong M, Li T, Chen L, Li T, Surgical outcomes in patients with locally advanced gastric cancer treated with S-1 and oxaliplatin as neoadjuvant chemotherapy. World J Surg Oncol. 2015;13:11

25. Xing J, Wang Y, Shan F, Li S, Jia Y, Ying X, et al. Comparison of totally laparoscopic and laparoscopic assisted gastrectomy after neoadjuvant chemotherapy in locally advanced gastric cancer. Eur $\mathrm{J}$ Surg Oncol. 2021;6:0748-7983.

26. Li Z, Shan F, Ying X, Zhang Y, Wang Y, Ren H, et al. Assessment of Laparoscopic Distal Gastrectomy After Neoadjuvant Chemotherapy for Locally Advanced Gastric Cancer: A Randomized Clinical Trial. JAMA Surg. 2019;154:1093-1101. 